\title{
On the Preliminary Test Generalized Liu Estimator with Series of Stochastic Restrictions
}

\author{
Mohammad H. Karbalaee ${ }^{1}$, Seyed Mohammad M. Tabatabaey ${ }^{2}$, and Mohammad \\ Arashi $^{3}$ \\ ${ }^{1}$ Department of Statistics, Ferdowsi University of Mashhad, International campus, Iran. \\ ${ }^{2}$ Department of Statistics, Ferdowsi University of Mashhad, Mashhad, Iran. \\ ${ }^{3}$ Department of Statistics, Shahrood University of Technology, Shahrood, Iran.
}

Received: 12/07/2017, Revision received: 03/05/2018, Published online: 03/28/2019

\begin{abstract}
When a series of stochastic restrictions are available, we study the performance of the preliminary test generalized Liu estimators (PTGLEs) based on the Wald, likelihood ratio and Lagrangian multiplier tests. In this respect, the optimal range of the biasing parameter is obtained under the mean square error sense. For this, the minimum/maximum value of the biasing matrix components is used to give the proper optimal range, where the biasing matrix is $D=\operatorname{diag}\left(d_{1}, d_{2}, \ldots, d_{p}\right), 0<d_{i}<1$, $i=1, \ldots, p$. We support our findings by some numerical illustrations.
\end{abstract}

Keywords. Biasing matrix, Generalized Liu estimator, Multiple regression model, Preliminary test estimator, Stochastic restriction.

MSC: 62J07; 62F15; 62F03; 62H05.

Mohammad H. Karbalaee (mhk70943000@yahoo.com)

Corresponding Author: Mohammad Arashi (m_arashi_stat@yahoo.com) 


\section{Introduction}

The multiple regression model (MRM) is arguably the most widely used statistical tools applied in almost every discipline of the modern era, however, in reality, there always exists some level of multicollinearity between the columns of design matrix that makes the estimation problem challenging. In this respect, the shrinkage estimation methodologies such as the ridge and Liu regressions are used as remedies. Biasedness of the resulting estimators sacrifices the efficiency. One method of improving the efficiency is to incorporate some level of prior information in the estimation problem. In this study, we only concentrate on the stochastic restriction as prior information. Stochastic restriction occurs in economics, microeconomics and financial studies. By using stochastic restriction, we can accomplish an examination and analysis of one's own thoughts and feelings (prior information via introspection). Also one may have prior information from a previous sample, which usually leads to obtaining some relations through stochastic subspace restrictions. Furthermore, combining stochastic restrictions with Zellner (1962) "Seemingly Unrelated" estimators, we can demonstrate good performance of the estimators using mean squared error (MSE) criterion comparatively. We refer to Ozkale (2009), Yang et al. (2009), Li and Yang (2010), Yang and Xu (2011), Yang and Wu (2012), Kuran and Ozkale (2016) to mention a few interesting and important studies in regression analysis with stochastic restriction.

Now, consider the following linear model

$$
y_{0}=X \beta+e,
$$

where $y_{0}=\left(y_{1}, y_{2}, \ldots, y_{n}\right)^{T}$ is an $n \times 1$ vector of observations on the dependent variable, $X$ is an $n \times p$ fixed matrix of full rank $p, \beta=\left(\beta_{1}, \beta_{2}, \ldots, \beta_{p}\right)^{T}$ is a $p \times 1$ vector of unknown parameters and $e=\left(e_{1}, e_{2}, \ldots, e_{n}\right)^{T}$ is an $n \times 1$ vector of errors, which is distributed according to the $n$-variate $\mathrm{t}$-distribution with $E(e)=0$ and $E\left(e e^{T}\right)=\sigma_{e}^{2} V_{1}$, where $V_{1}$ is an $n \times n$ known positive definite symmetric matrix and $\sigma_{e}^{2}=v \sigma^{2} /(v-2), \sigma^{2} \in R^{+}$is unknown, with $v$ degree of freedom and probability density function

$$
f(e)=\frac{\Gamma\left(\frac{n+v}{2}\right)\left|V_{1}\right|^{-\frac{1}{2}}}{(\pi v)^{\frac{n}{2}} \Gamma\left(\frac{v}{2}\right) \sigma^{n}}\left(1+\frac{e^{T} V_{1} e}{v \sigma^{2}}\right)^{-\frac{n+v}{2}}, \quad 0<v, \sigma<\infty .
$$

We designate $e \sim \mathcal{T}_{n}\left(\mathbf{0}, \sigma^{2} V_{1}, v\right)$.

Among several researchers, Kibria (1996), Khan and Saleh (1997), Kibria and Saleh (2004) and Tabatabaey et al. (2004) have well motivated to use multivariate t-distribution in linear regression models. 
It is well known that the generalized least squares (GLS) estimator of $\beta$ is given by $\tilde{\beta}=C^{-1} X^{T} V_{1}^{-1} y_{0}$ with $C=X^{T} V_{1}^{-1} X$. Under the exact restriction $r=R \beta$, where $r$ is an $q \times 1$ vector of known elements and $R$ is a $q \times p$ known prior information matrix of rank $q \leq p$, the restricted GLS (RGLS) estimator of $\beta$ is given by

$$
\hat{\beta}=\tilde{\beta}-C^{-1} R^{T}\left(R C^{-1} R^{T}\right)^{-1}(R \tilde{\beta}-r) .
$$

The RGLSE performs better than the GLSE, when the non-stochastic restriction $r=R \beta$ holds, but as the parameter, $\beta$ moves away from the subspace $r=R \beta$, the RGLSE becomes biased and inefficient while the performance of the GLSE remains stable. In order to corporate the information existing in both the GLSE and RGSLE, one may combine these estimators to achieve a better estimator in the risk sense. This gives rise to the definition of preliminary test GLS (PTGLS) estimator with form

$$
\hat{\beta}^{P T}=\hat{\beta} I\left(L_{*} \leq L_{n, \alpha}\right)+\tilde{\beta} I\left(L_{*}>L_{n, \alpha}\right),
$$

where $L_{*}$ is a general test statistics for testing the hypothesis $H_{0}: r=R \beta, L_{n, \alpha}$ is the $\alpha$ level critical value of $L_{*}$ and $I(A)$ is the indicator function of the set $A$. The idea of the PTGLSE was first proposed by Bancroft (1944). Afterward, researchers endeavored to develop preliminary test estimators in different fields of statistical inference. Among others, we refer to the studies by Saleh and Sen (1978), Saleh (2006), Roozbeh (2015) and references therein, and more recently Yuzbasi et al. (2017), Wu and Asar (2017), and Yuzbasi et al. (2018).

For the ill-conditioned design matrix $X^{T} X$, under a multicollinear situation, Yang and $\mathrm{Xu}$ (2011) proposed Liu-type of RGLSE and PTGLE in the t-regression model with stochastic restriction. Indeed, they proposed to use Liu's estimator (1993) given by

$$
\tilde{\beta}(d)=\left(C+I_{p}\right)^{-1}\left(X^{T} V_{1}^{-1} y_{0}+d \tilde{\beta}\right)=F_{d} \tilde{\beta}, \quad F_{d}=\left(C+I_{p}\right)^{-1}\left(C+d I_{p}\right), \quad 0<d<1 .
$$

to construct Liu-type RGLSE and PTGLSE by premultiplying them using $F_{d}$.

The purpose of our study is to follow the work of Yang and Xu (2009) and construct Liu-type preliminary test estimator. However, we have some other concerns:

1. What is the optimal range for the biasing parameter, in the MSE sense, when one uses the generalized Liu estimator (GLE) of Akdeniz and Kaciranlar (1995) given by

$$
\tilde{\beta}(D)=\left(C+I_{p}\right)^{-1}\left(X^{T} V_{1}^{-1} y_{0}+D \tilde{\beta}\right)=F_{D} \tilde{\beta},
$$

where $F_{D}=\left(C+I_{p}\right)^{-1}(C+D)$ and $D=\operatorname{diag}\left(d_{1}, d_{2}, \ldots, d_{p}\right)$ is the biasing matrix, $0<d_{i}<1, i=1, \ldots, p$ ? 
2. Is it possible to have different sources of prior information?

The first question above will be covered in the comparison of MSE functions of the generalized Liu preliminary test estimators. Our proposal to answer the second question is to use a series of stochastic restrictions. Very recently Karbalaee et al. (2017), used a series of stochastic restrictions to obtain preliminary test estimators in ridge regression (RR) under the normality assumption for the error term. However, a drawback of RR method is its non-linearity relation with respect to the ridge parameter, which cannot be estimated in a closed form and hence optimized. Here we use a different approach to combat this drawback.

We organize our paper as follows. The generalized Liu preliminary test estimators based on the Wald (W), the likelihood ratio (LR) and Lagrangian multiplier (LM) tests are proposed in Section 2, while their properties are obtained in Section 3. Section 4 contains the relative performance of the estimators. The estimation of the biasing matrix $D$ is the content of Section 5. Numerical illustrations are provided in Section 6. The paper is concluded in Section 7.

\section{Series of Stochastic Restrictions}

If uncertainty exists about the prior information specifications, one alternative is to make use of stochastic linear restrictions. Stochastic restriction arises from prior statistical information, usually in the form of previous estimates of parameters, and take the form of an additional linear model.

Consider a series of stochastic restrictions about $\beta$ with forms

$$
\left\{\begin{array}{c}
r_{1}=R_{1} \beta+\gamma_{1} \\
r_{2}=R_{2} \beta+\gamma_{2} \\
\vdots \\
r_{l}=R_{l} \beta+\gamma_{l},
\end{array}\right.
$$

where $r_{i}$ is a known $q_{i} \times 1$ random vector, $R_{i}$ is a known $q_{i} \times p$ prior information of rank $q_{i} \leq p$ and $\gamma_{i}$ is a random vector with $\gamma_{i} \sim \mathcal{T}_{q_{i}}\left(\mu_{i}, \sigma^{2} V_{2}^{(i)}, v\right)$, where $V_{2}^{(i)}$ is a $q_{i} \times q_{i}$ known positive definite symmetric matrix, with $\sum_{j=1}^{l} q_{j}=q$. We further assume that $e_{i}$ and $\gamma_{i}$, $i=1, \ldots, l$ are independent. 
Combining the linear model (1.1) and stochastic restrictions (2.1), we obtain the following mixed model

$$
Y=Z \phi+\varepsilon
$$

with prior information

$$
H_{0}: H \phi=\mu=0,
$$

where

$$
\begin{array}{r}
Y=\left(y_{0}, r_{1}, \ldots, r_{l}\right)^{T}, \quad Z=\left[\begin{array}{cc}
X_{n \times p} & 0_{n \times q} \\
0_{q \times p} & I_{q}
\end{array}\right], \quad \phi=\left[\begin{array}{c}
\beta \\
R \beta+\mu
\end{array}\right], \\
\mu=\left(\mu_{1}, \ldots, \mu_{l}\right)^{T}, \quad \varepsilon=\left(e^{T}, \gamma^{T}-\mu^{T}\right)^{T},
\end{array} \begin{array}{r}
\gamma=\left(\gamma_{1}, \ldots, \gamma_{l}\right)^{T}, \\
H=\left[\begin{array}{ll}
-R & I_{q}
\end{array}\right], \quad R=\left(R_{1}^{T}, \ldots, R_{l}^{T}\right)^{T} .
\end{array}
$$

The distribution of the error term $\varepsilon$ is $\mathcal{T}_{n+q}\left(\left(0^{T}, \mu^{T}\right)^{T}, \sigma^{2} V, v\right)$, where $V=\operatorname{diag}\left(V_{1}, V_{2}^{(1)}, \ldots, V_{2}^{(l)}\right)$.

Then, the GLE and RGLE of $\phi$ are respectively given by

$$
\begin{aligned}
& \tilde{\phi}(D)=F_{D} \tilde{\phi}=\left(C+I_{p+q}\right)^{-1}\left(Z^{T} V^{-1} Y+D \tilde{\phi}\right), \\
& \hat{\phi}(D)=F_{D} \hat{\phi}=F_{D} \tilde{\phi}-F_{D} C^{-1} H^{T}\left(H C^{-1} H^{T}\right)^{-1} H \tilde{\phi},
\end{aligned}
$$

where $F_{D}=\left(C+I_{p+q}\right)^{-1}(C+D)$ and $C=Z^{T} V^{-1} Z$.

To test the null-hypothesis $H_{0}: \mu=0$, with $\mathrm{t}$-distributed errors, we may use one of the three general test statistics W, LR and LM. These are given by

$$
\begin{aligned}
L_{W} & =\frac{\lambda(n)(H \tilde{\phi})^{T}\left(H C^{-1} H^{T}\right)^{-1} H \tilde{\phi}}{\tilde{\sigma}^{2}}, \\
L_{L R} & =(n+q)\left(L n \hat{\sigma}^{2}-L n \tilde{\sigma}^{2}\right), \\
L_{L M} & =\frac{(H \tilde{\phi})^{T}\left(H C^{-1} H^{T}\right)^{-1} H \tilde{\phi}}{\lambda(n) \hat{\sigma}^{2}},
\end{aligned}
$$

where

$$
\tilde{\sigma}^{2}=\frac{(Y-Z \tilde{\phi})^{T} V^{-1}(Y-Z \tilde{\phi})}{n+q}, \quad \text { and } \quad \hat{\sigma}^{2}=\frac{(Y-Z \hat{\phi})^{T} V^{-1}(Y-Z \hat{\phi})}{n+q}
$$


are the unrestricted and restricted maximum likelihood estimators of $\sigma^{2}$ and

$$
\lambda(n)=\frac{n+q+v}{n+q+v+2}, \quad 0<\lambda(n)<1 .
$$

For more details, we refer to Ullah and Walsh (1984). Note that if $n$ is large then $\lambda(n)$ is close to 1 , then the results in the above equations also hold for the normal regression model.

We propose the PTGL estimators under stochastic constraints, based on the W, LR and LM test statistics, respectively as

$$
\begin{aligned}
& \hat{\phi}_{W}^{P T G L}(D)=\hat{\phi}(D) I\left(L_{W} \leq \chi_{q}^{2}(\alpha)\right)+\tilde{\phi}(D) I\left(L_{W}>\chi_{q}^{2}(\alpha)\right), \\
& \hat{\phi}_{L R}^{P T G L}(D)=\hat{\phi}(D) I\left(L_{L R} \leq \chi_{q}^{2}(\alpha)\right)+\tilde{\phi}(D) I\left(L_{L R}>\chi_{q}^{2}(\alpha)\right), \\
& \hat{\phi}_{L M}^{P T G L}(D)=\hat{\phi}(D) I\left(L_{L M} \leq \chi_{q}^{2}(\alpha)\right)+\tilde{\phi}(D) I\left(L_{L M}>\chi_{q}^{2}(\alpha)\right),
\end{aligned}
$$

where $\chi_{q}^{2}(\alpha)$ denotes the upper $\alpha$ critical value of chi-square distribution with q degree of freedom.

\section{Properties}

The bias and MSE expressions of the proposed generalized Liu estimators of $\phi$ are discussed in this section. The bias and MSE are evaluated by $\mathrm{B}(\hat{\theta})=\mathbb{E}(\hat{\theta})-\theta$ and $\operatorname{MSE}(\hat{\theta})=\mathbb{E}(\hat{\theta}-\theta)^{T}(\hat{\theta}-\theta)$, for any estimator $\hat{\theta}$ of $\theta$.

Theorem 3.1. The bias expressions of the GL, RGL and PTGL estimators are given by

$$
\begin{aligned}
& \operatorname{Bias}(\tilde{\phi}(D))=-(C+I)^{-1}(I-D) \phi, \quad \operatorname{Bias}(\hat{\phi}(D))=-(C+I)^{-1}(I-D) \phi-F_{D} \delta, \\
& \operatorname{Bias}\left(\hat{\phi}_{W}^{P T}(D)\right)=-(C+I)^{-1}(I-D) \phi-F_{D} \delta G_{q+2, m}^{(2)}\left(x_{1}^{W}, \Delta\right), \\
& \operatorname{Bias}\left(\hat{\phi}_{L R}^{P T}(D)\right)=-(C+I)^{-1}(I-D) \phi-F_{D} \delta G_{q+2, m}^{(2)}\left(x_{1}^{L R}, \Delta\right),
\end{aligned}
$$




$$
\operatorname{Bias}\left(\hat{\phi}_{L M}^{P T}(D)\right)=-(C+I)^{-1}(I-D) \phi-F_{D} \delta G_{q+2, m}^{(2)}\left(x_{1}^{L M}, \Delta\right)
$$

where $\delta=C^{-1} H^{T}\left(H C^{-1} H^{T}\right)^{-1} H \phi$,

$$
x_{1}^{W}=\frac{m \chi_{q}^{2}(\alpha)}{\lambda(n)(n+q)(q+2)}, \quad x_{1}^{L R}=\frac{m}{q+2}\left(e^{\frac{x_{q}^{2}(\alpha)}{n+q}}-1\right), \quad x_{1}^{L M}=\frac{m \lambda(n) \chi_{q}^{2}(\alpha)}{(q+2)\left(n+q-\lambda(n) \chi_{q}^{2}(\alpha)\right)},
$$

and $G_{q+2, m}^{(2)}\left(x^{*}, \Delta\right)$ is defined by the following function

$$
G_{q+2 i, m}^{(j)}\left(x^{*}, \Delta\right)=\sum_{r=0}^{\infty} \frac{\Gamma\left(\frac{v}{2}+r+j-2\right)}{\Gamma(r+1) \Gamma\left(\frac{v}{2}+j-2\right)} \frac{\left(\frac{\Delta}{v-2}\right)^{r}}{\left(1+\frac{\Delta}{v-2}\right)^{\frac{v}{2}+r+j-2}} I_{x^{*}}\left\{\frac{q+2 i}{2}+r, \frac{m}{2}\right\},
$$

where $I_{x^{*}}\{. .$.$\} is the incomplete beta function and x^{*}$ denotes any of the quantities, $x_{1}^{W}, x_{1}^{L R}$ and $x_{1}^{L M}$ and $\Delta=\frac{(H \phi)^{T}\left(H C^{-1} H^{T}\right)^{-1} H \phi}{\sigma_{e}^{2}}$.

Theorem 3.2. The MSE expressions of the GL, RGL and PTGL estimators are given by

$$
\begin{aligned}
\operatorname{MSE}(\tilde{\phi}(D))= & \sigma_{e}^{2} \operatorname{tr}\left(F_{D} C^{-1} F_{D}^{T}\right)+\phi^{T}(I-D)^{T}(C+I)^{-2}(I-D) \phi, \\
\operatorname{MSE}(\hat{\phi}(D))= & \sigma_{e}^{2} \operatorname{tr}\left(F_{D} C^{-1} F_{D}\right)-\sigma_{e}^{2} \operatorname{tr}\left[F_{D} C^{-1} H^{T}\left(H C^{-1} H^{T}\right)^{-1} H C^{-1} F_{D}^{T}\right] \\
& +\delta^{T} F_{D}^{T} F_{D} \delta+2 \delta^{T} F_{D}^{T}(I-D)(C+I)^{-1} \phi+\phi^{T}(I-D)^{T}(C+I)^{-2}(I-D) \phi,
\end{aligned}
$$

$\operatorname{MSE}\left(\hat{\phi}_{W}^{P T G L}(D)\right)=\sigma_{e}^{2} \operatorname{tr}\left(F_{D} C^{-1} F_{D}^{T}\right)-\sigma_{e}^{2} \operatorname{tr}\left[F_{D} C^{-1} H^{T}\left(H C^{-1} H^{T}\right)^{-1} H C^{-1} F_{D}^{T}\right] G_{q+2, m}^{(1)}\left(x_{1}^{W}, \Delta\right)$ $+\delta^{T} F_{D}^{T} F_{D} \delta\left[2 G_{q+2, m}^{(2)}\left(x_{1}^{W}, \Delta\right)-G_{q+4, m}^{(2)}\left(x_{2}^{W}, \Delta\right)\right]$ $+2 \delta^{T} F_{D}^{T}(I-D)(C+I)^{-1} \phi G_{q+2, m}^{(2)}\left(x_{1}^{W}, \Delta\right)+\phi^{T}(I-D)(C+I)^{-2}(I-D) \phi$,

$\operatorname{MSE}\left(\hat{\phi}_{L R}^{P T G L}(D)\right)=\sigma_{e}^{2} \operatorname{tr}\left(F_{D} C^{-1} F_{D}^{T}\right)-\sigma_{e}^{2} \operatorname{tr}\left[F_{D} C^{-1} H^{T}\left(H C^{-1} H^{T}\right)^{-1} H C^{-1} F_{D}^{T}\right] G_{q+2, m}^{(1)}\left(x_{1}^{L R}, \Delta\right)$ 


$$
\begin{aligned}
& +\delta^{T} F_{D}^{T} F_{D} \delta\left[2 G_{q+2, m}^{(2)}\left(x_{1}^{L R}, \Delta\right)-G_{q+4, m}^{(2)}\left(x_{2}^{L R}, \Delta\right)\right] \\
& +2 \delta^{T} F_{D}^{T}(I-D)(C+I)^{-1} \phi G_{q+2, m}^{(2)}\left(x_{1}^{L R}, \Delta\right)+\phi^{T}(I-D)(C+I)^{-2}(I-D) \phi,
\end{aligned}
$$

$$
\begin{aligned}
\operatorname{MSE}\left(\hat{\phi}_{L M}^{P T G L}(D)\right)= & \sigma_{e}^{2} \operatorname{tr}\left(F_{D} C^{-1} F_{D}^{T}\right)-\sigma_{e}^{2} \operatorname{tr}\left[F_{D} C^{-1} H^{T}\left(H C^{-1} H^{T}\right)^{-1} H C^{-1} F_{D}^{T}\right] G_{q+2, m}^{(1)}\left(x_{1}^{L M}, \Delta\right) \\
& +\delta^{T} F_{D}^{T} F_{D} \delta\left[2 G_{q+2, m}^{(2)}\left(x_{1}^{L M}, \Delta\right)-G_{q+4, m}^{(2)}\left(x_{2}^{L M}, \Delta\right)\right] \\
& +2 \delta^{T} F_{D}^{T}(I-D)(C+I)^{-1} \phi G_{q+2, m}^{(2)}\left(x_{1}^{L M}, \Delta\right)+\phi^{T}(I-D)(C+I)^{-2}(I-D) \phi,
\end{aligned}
$$

where

$$
x_{2}^{W}=\frac{m \chi_{q}^{2}(\alpha)}{\lambda(n)(n+q)(q+4)}, \quad x_{2}^{L R}=\frac{m}{q+4}\left(e^{\frac{\chi_{q}^{2}(\alpha)}{n+q}}-1\right), \quad x_{2}^{L M}=\frac{m \lambda(n) \chi_{q}^{2}(\alpha)}{(q+4)\left(n+q-\lambda(n) \chi_{q}^{2}(\alpha)\right)},
$$

and $G_{q+2, m}^{(1)}(. ; \Delta), G_{q+4, m}^{(2)}(. ; \Delta)$ is a function according to equation (3.1).

Proofs of the above theorems are similar to the ones in Yang and $\mathrm{Xu}$ (2011).

\section{Performance Analysis}

Comparison of relative performance of the proposed estimators as a function of $\Delta$ and $D$ is presented in this section.

Using the spectral decomposition of $C$ as $C=\Gamma \Lambda \Gamma^{T}$, with $\Lambda=\operatorname{diag}\left(\lambda_{1}, \lambda_{2}, \ldots, \lambda_{p+q}\right)$, such that $\lambda_{1} \geq \lambda_{2} \geq \ldots \geq \lambda_{p+q}>0$, it is easy to see that eigenvalues of $C+I_{p+q}$ and $F_{D}$ are equal to $\lambda_{i}+1$ and $\left(\lambda_{i}+d_{i}\right) /\left(\lambda_{i}+1\right)$, respectively. In order to use the results of Theorems $3.1 \& 3.2$, we consider the following equalities based on the eigenvalues of $C$ :

$$
\begin{aligned}
\phi^{T}(I-D)^{T}(C+I)^{-2}(I-D) \phi & =\sum_{i=1}^{p+q} \frac{\phi_{i}^{*^{2}}\left(1-d_{i}\right)^{2}}{\left(\lambda_{i}+1\right)^{2}} \\
\delta^{T} F_{D}^{T}(I-D)(C+I)^{-1} \phi & =\sum_{i=1}^{p+q} \frac{\left(\lambda_{i}+d_{i}\right)\left(1-d_{i}\right) \delta_{i}^{*} \phi_{i}^{*}}{\left(\lambda_{i}+1\right)^{2}}, \quad \phi^{*}=\Gamma^{T} \phi, \quad \delta^{*}=\Gamma^{T} \delta, \\
\operatorname{tr}\left(F_{D}^{T} C^{-1} F_{D}\right) & =\sum_{i=1}^{p+q} \frac{\left(\lambda_{i}+d_{i}\right)^{2}}{\lambda_{i}\left(\lambda_{i}+1\right)^{2}}
\end{aligned}
$$




$$
\begin{aligned}
\delta^{T} F_{D}^{T} F_{D} \delta & =\sum_{i=1}^{p+q} \frac{\delta_{i}^{*^{2}}\left(\lambda_{i}+d_{i}\right)^{2}}{\left(\lambda_{i}+1\right)^{2}} \\
\operatorname{tr}\left(F_{D} A F_{D}^{T}\right) & =\sum_{i=1}^{p+q} \frac{a_{i i}^{*}\left(\lambda_{i}+d_{i}\right)^{2}}{\left(\lambda_{i}+1\right)^{2}}
\end{aligned}
$$

where $a_{i i}^{*} \geq 0$ is the $i$ th diagonal element of the matrix $A^{*}=\Gamma^{T} A \Gamma, A=C^{-1} H^{T}\left(H C^{-1} H^{T}\right)^{-1} H C^{-1}$.

Note that by Courant Theorem (see Theorem A.2.4 of Anderson (2003)), we have

$$
\frac{\delta^{T} F_{D}^{T} F_{D} \delta}{\delta^{T} \delta} \leq \gamma_{\max }\left(F_{D}^{T} F_{D}\right)=\gamma_{1}
$$

Hence,

$$
\delta^{T} F_{D}^{T} F_{D} \delta \leq \gamma_{1} \delta^{T} \delta=\sigma_{e}^{2} \Delta \gamma_{1}
$$

Similarly, using the smallest eigenvalue we have

$$
\sigma_{e}^{2} \Delta \gamma_{p+q} \leq \delta^{T} F_{D}^{T} F_{D} \delta
$$

where $\gamma_{1}$ and $\gamma_{p+q}$ are the largest and smallest eigenvalues of $F_{D}^{T} F_{D}$.

In order to have a scalar measurement for the bias, we define the quadratic bias as

$$
Q B_{*}(D, \alpha, \Delta)=B_{*}^{T}(D, \alpha, \Delta) B_{*}(D, \alpha, \Delta),
$$

where $B_{*}($.$) defines for the bias function either of the \tilde{\phi}_{(D)}, \hat{\phi}_{(D)}, \hat{\phi}_{W}^{P T G L}(D), \hat{\phi}_{L R}^{P T G L}(D)$ and $\hat{\phi}_{L M}^{P T G L}(D)$. The following conclusion can be proved similar to Kibria and Saleh (2004).

Proposition 4.1. For all values of $d_{i} \in(0,1), \alpha$ and $\Delta$ we have a relation between the quadratic biases of the PTGL estimators, given by

$$
Q B_{W}(D, \alpha, \Delta) \leq Q B_{L R}(D, \alpha, \Delta) \leq Q B_{L M}(D, \alpha, \Delta) .
$$

Assuming $D$ and $\alpha$ are known, we compare the MSE of the PTGLEs based on three tests as a function of $\Delta$ and determine their dominance properties. Consider

$$
\begin{aligned}
\Upsilon_{1}=\operatorname{MSE}\left(\hat{\phi}_{W}^{P T G L}(D)\right)-\operatorname{MSE}\left(\hat{\phi}_{L R}^{P T G L}(D)\right)=[ & \left.\sigma_{e}^{2} \operatorname{tr}\left(F_{D} A F_{D}^{T}\right)-2 \phi^{T}(C+I)^{-1}(I-D) F_{D} \delta\right] \mathcal{H} \\
& -\delta^{T} F_{D}^{T} F_{D} \delta(2 \mathcal{H}-\mathcal{E}),
\end{aligned}
$$


where $\mathcal{H}=G_{q+2, m}^{(2)}\left(x_{1}^{L R}, \Delta\right)-G_{q+2, m}^{(2)}\left(x_{1}^{W}, \Delta\right) \quad$ and $\quad \mathcal{E}=G_{q+4, m}^{(2)}\left(x_{2}^{L R}, \Delta\right)-G_{q+4, m}^{(2)}\left(x_{2}^{W}, \Delta\right)$. Since for all $n, p, q, \lambda(n)$ and $\alpha$ we have $x_{1}^{W} \leq x_{1}^{L R} \leq x_{1}^{L M}$ and $x_{2}^{W} \leq x_{2}^{L R} \leq x_{2}^{L M}$, hence $\mathcal{H}$ and $\mathcal{E}$ are positive for all $d_{i}, \Delta$ and $\alpha$ and $\Upsilon_{1}$ is non-negative, i.e., $\hat{\phi}_{L R}^{P T G L}(D)$ is better than $\hat{\phi}_{W}^{P T G L}(D)$, if and only if

$$
0<\Delta \leq \frac{\left[\operatorname{tr}\left(F_{D} A F_{D}^{T}\right)-2 \sigma_{e}^{-2} \phi^{T}(C+I)^{-1}(I-D) F_{D} \delta\right] \mathcal{H}}{\gamma_{1}(2 \mathcal{H}-\mathcal{E})}=\Delta_{1}(D, \alpha),
$$

where

$$
\Delta_{1}(D, \alpha)=\frac{\mathcal{H}}{\gamma_{1}(2 \mathcal{H}-\mathcal{E})} \sum_{i=1}^{p+q} \frac{\left(\lambda_{i}+d_{i}\right)\left(a_{i i}^{*}\left(\lambda_{i}+d_{i}\right)-2 \sigma_{e}^{-2} \delta_{i}^{*} \phi_{i}^{*}\left(1-d_{i}\right)\right)}{\left(\lambda_{i}+1\right)^{2}}
$$

Similarly, $\hat{\phi}_{W}^{P T G L}(D)$ is better than $\hat{\phi}_{L R}^{P T G L}(D)$, whenever

$$
\Delta>\frac{\mathcal{H}}{\gamma_{p+q}(2 \mathcal{H}-\mathcal{E})} \sum_{i=1}^{p+q} \frac{\left(\lambda_{i}+d_{i}\right)\left(a_{i i}^{*}\left(\lambda_{i}+d_{i}\right)-2 \sigma_{e}^{-2} \delta_{i}^{*} \phi_{i}^{*}\left(1-d_{i}\right)\right)}{\left(\lambda_{i}+1\right)^{2}}=\Delta_{2}(D, \alpha) .
$$

Under the null-hypothesis (2.3), $\hat{\phi}_{L R}^{P T G L}(D)$ is always better than $\hat{\phi}_{W}^{P T G L}(D)$.

Now, for the MSE comparison of $\hat{\phi}_{L R}^{P T G L}(D)$ and $\hat{\phi}_{L M}^{P T G L}(D)$, consider the MSE difference

$$
\begin{gathered}
\Upsilon_{2}=\operatorname{MSE}\left(\hat{\phi}_{L R}^{P T G L}(D)\right)-\operatorname{MSE}\left(\hat{\phi}_{L M}^{P T G L}(D)\right)=\left[\sigma_{e}^{2} \operatorname{tr}\left(F_{D} A F_{D}^{T}\right)-2 \phi^{T}(C+I)^{-1}(I-D) F_{D} \delta\right] \mathcal{H}^{*} \\
-\delta^{T} F_{D}^{T} F_{D} \delta\left(2 \mathcal{H}^{*}-\mathcal{E}^{*}\right),
\end{gathered}
$$

where $\mathcal{H}^{*}=G_{q+2, m}^{(2)}\left(x_{1}^{L M}, \Delta\right)-G_{q+2, m}^{(2)}\left(x_{1}^{L R}, \Delta\right)$ and $\mathcal{E}^{*}=G_{q+4, m}^{(2)}\left(x_{2}^{L M}, \Delta\right)-G_{q+4, m}^{(2)}\left(x_{2}^{L R}, \Delta\right)$. Since $\mathcal{H}^{*}$ and $\mathcal{E}^{*}$ are positive for all $d_{i}, \Delta$ and $\alpha, \Upsilon_{2}$ is non-negative, i.e., $\hat{\phi}_{L M}^{P T G L}(D)$ is better than $\hat{\phi}_{L R}^{P T G L}(D)$, if and only if

$$
0 \leq \Delta \leq \frac{\mathcal{H}^{*}}{\gamma_{1}\left(2 \mathcal{H}^{*}-\mathcal{E}^{*}\right)} \sum_{i=1}^{p+q} \frac{\left(\lambda_{i}+d_{i}\right)\left(a_{i i}^{*}\left(\lambda_{i}+d_{i}\right)-2 \sigma_{e}^{-2} \delta_{i}^{*} \phi_{i}^{*}\left(1-d_{i}\right)\right)}{\left(\lambda_{i}+1\right)^{2}}=\Delta_{3}(D, \alpha) .
$$

Also $\hat{\phi}_{L R}^{P T G L}(D)$ is better than $\hat{\phi}_{L M}^{P T G L}(D)$, whenever

$$
\Delta>\frac{\mathcal{H}^{*}}{\gamma_{p+q}\left(2 \mathcal{H}^{*}-\mathcal{E}^{*}\right)} \sum_{i=1}^{p+q} \frac{\left(\lambda_{i}+d_{i}\right)\left(a_{i i}^{*}\left(\lambda_{i}+d_{i}\right)-2 \sigma_{e}^{-2} \delta_{i}^{*} \phi_{i}^{*}\left(1-d_{i}\right)\right)}{\left(\lambda_{i}+1\right)^{2}}=\Delta_{4}(D, \alpha) .
$$


Under the null-hypothesis (2.3), $\hat{\phi}_{L M}^{P T G L}(D)$ is always better than $\hat{\phi}_{L R}^{P T G L}(D)$. Hence, we have the following result:

Proposition 4.2. Under the null-hypothesis (2.3), the MSE of the PTGLE based on W, LR and LM tests satisfies the following dominance relationship

$$
\operatorname{MSE}\left(\hat{\phi}_{L M}^{P T G L}(D)\right) \leq \operatorname{MSE}\left(\hat{\phi}_{L R}^{P T G L}(D)\right) \leq \operatorname{MSE}\left(\hat{\phi}_{W}^{P T G L}(D)\right) .
$$

Under the alternative hypothesis, we have

$$
\begin{aligned}
& \operatorname{MSE}\left(\hat{\phi}_{L M}^{P T G L}(D)\right) \leq \operatorname{MSE}\left(\hat{\phi}_{L R}^{P T G L}(D)\right) \leq \operatorname{MSE}\left(\hat{\phi}_{W}^{P T G L}(D)\right) \quad \text { if } \quad \Delta \in\left[0, \Delta_{13}(D, \alpha)\right] \text {, } \\
& \operatorname{MSE}\left(\hat{\phi}_{W}^{P T G L}(D)\right) \leq \operatorname{MSE}\left(\hat{\phi}_{L R}^{P T G L}(D)\right) \leq \operatorname{MSE}\left(\hat{\phi}_{L M}^{P T G L}(D)\right) \quad \text { if } \quad \Delta \in\left(\Delta_{24}(D, \alpha), \infty\right) \text {, } \\
& \text { where } \Delta_{13}(D, \alpha)=\min \left\{\Delta_{1}(D, \alpha), \Delta_{3}(D, \alpha)\right\} \text { and } \Delta_{24}(D, \alpha)=\max \left\{\Delta_{2}(D, \alpha), \Delta_{4}(D, \alpha)\right\} \text {. }
\end{aligned}
$$

Now, we analyze the MSE of the PTGLEs based on three tests as a function of $D$ and determine their dominance properties. We have

$$
\Upsilon_{1}=\sum_{i=1}^{p+q} \frac{\left(\lambda_{i}+d_{i}\right)}{\left(\lambda_{i}+1\right)^{2}}\left[\sigma_{e}^{2} a_{i i}^{*} \lambda_{i} \mathcal{H}-2 \phi_{i}^{*} \delta_{i}^{*} \mathcal{H}-\delta_{i}^{*^{2}} \lambda_{i}(2 \mathcal{H}-\mathcal{E})+\left(\sigma_{e}^{2} a_{i i}^{*} \mathcal{H}+2 \phi_{i}^{*} \delta_{i}^{*} \mathcal{H}-\delta_{i}^{*^{2}}(2 \mathcal{H}-\mathcal{E})\right) d_{i}\right] .
$$

Considering $d_{\min }=\min _{1 \leq i \leq p+q}\left\{d_{i}\right\}$ and $d_{\max }=\max _{1 \leq i \leq p+q}\left\{d_{i}\right\}$ and

$$
d_{1}(\Delta, \alpha)=\frac{\max _{1 \leq i \leq p+q}\left\{(2 \mathcal{H}-\mathcal{E}) \delta_{i}^{*^{2}} \lambda_{i}+2 \mathcal{H} \phi_{i}^{*} \delta_{i}^{*}-\sigma_{e}^{2} \mathcal{H} a_{i i}^{*} \lambda_{i}\right\}}{\min _{1 \leq i \leq p+q}\left\{\sigma_{e}^{2} \mathcal{H} a_{i i}^{*}+2 \mathcal{H} \phi_{i}^{*} \delta_{i}^{*}-(2 \mathcal{H}-\mathcal{E}) \delta_{i}^{*^{2}}\right\}}
$$

and

$$
d_{2}(\Delta, \alpha)=\frac{\min _{1 \leq i \leq p+q}\left\{(2 \mathcal{H}-\mathcal{E}) \delta_{i}^{*^{2}} \lambda_{i}+2 \mathcal{H} \phi_{i}^{*} \delta_{i}^{*}-\sigma_{e}^{2} \mathcal{H} a_{i i}^{*} \lambda_{i}\right\}}{\max _{1 \leq i \leq p+q}\left\{\sigma_{e}^{2} \mathcal{H} a_{i i}^{*}+2 \mathcal{H} \phi_{i}^{*} \delta_{i}^{*}-(2 \mathcal{H}-\mathcal{E}) \delta_{i}^{*^{2}}\right\}}
$$

$\Upsilon_{1}$ is non-negative, i.e., $\hat{\phi}_{L R}^{P T G L}(D)$ is better than $\hat{\phi}_{W}^{P T G L}(D)$, if and only if $d_{1}(\Delta, \alpha) \leq d_{\text {min }}<1$ and $\hat{\phi}_{W}^{P T G L}(D)$ is better than $\hat{\phi}_{L R}^{P T G L}(D)$, if and only if $0<d_{\max } \leq d_{2}(\Delta, \alpha)$.

Now, for the MSE comparison of $\hat{\phi}_{L R}^{P T G L}(D)$ and $\hat{\phi}_{L M}^{P T G L}(D)$, we have $\Upsilon_{2}=\sum_{i=1}^{p+q} \frac{\left(\lambda_{i}+d_{i}\right)}{\left(\lambda_{i}+1\right)^{2}}\left[\sigma_{e}^{2} a_{i i}^{*} \lambda_{i} \mathcal{H}^{*}-2 \phi_{i}^{*} \delta_{i}^{*} \mathcal{H}^{*}-\delta_{i}^{*^{2}} \lambda_{i}\left(2 \mathcal{H}^{*}-\mathcal{E} *\right)+\left(\sigma_{e}^{2} a_{i i}^{*} \mathcal{H}^{*}+2 \phi_{i}^{*} \delta_{i}^{*} \mathcal{H}^{*}-\delta_{i}^{*^{2}}\left(2 \mathcal{H}^{*}-\mathcal{E}^{*}\right)\right) d_{i}\right]$ 
similarly considering

$$
d_{3}(\Delta, \alpha)=\frac{\max _{1 \leq i \leq p+q}\left\{\left(2 \mathcal{H}^{*}-\mathcal{E}^{*}\right) \delta_{i}^{*^{2}} \lambda_{i}+2 \mathcal{H}^{*} \phi_{i}^{*} \delta_{i}^{*}-\sigma_{e}^{2} \mathcal{H}^{*} a_{i i}^{*} \lambda_{i}\right\}}{\min _{1 \leq i \leq p+q}\left\{\sigma_{e}^{2} \mathcal{H}^{*} a_{i i}^{*}+2 \mathcal{H}^{*} \phi_{i}^{*} \delta_{i}^{*}-\left(2 \mathcal{H}^{*}-\mathcal{E}^{*}\right) \delta_{i}^{*^{2}}\right\}},
$$

and

$$
d_{4}(\Delta, \alpha)=\frac{\min _{1 \leq i \leq p+q}\left\{\left(2 \mathcal{H}^{*}-\mathcal{E}^{*}\right) \delta_{i}^{*^{2}} \lambda_{i}+2 \mathcal{H}^{*} \phi_{i}^{*} \delta_{i}^{*}-\sigma_{e}^{2} \mathcal{H}^{*} a_{i i}^{*} \lambda_{i}\right\}}{\max _{1 \leq i \leq p+q}\left\{\sigma_{e}^{2} \mathcal{H}^{*} a_{i i}^{*}+2 \mathcal{H}^{*} \phi_{i}^{*} \delta_{i}^{*}-\left(2 \mathcal{H}^{*}-\mathcal{E}^{*}\right) \delta_{i}^{*^{2}}\right\}}
$$

$\Upsilon_{2}$ is non-negative, i.e., $\hat{\phi}_{L M}^{P T G L}(D)$ is better than $\hat{\phi}_{L R}^{P T G L}(D)$, if and only if $d_{3}(\Delta, \alpha) \leq d_{\text {min }}<1$ and $\hat{\phi}_{L R}^{P T G L}(D)$ is better than $\hat{\phi}_{L M}^{P T G L}(D)$, if and only if $0<d_{\max } \leq d_{4}(\Delta, \alpha)$.

Hence, we have the following result

Proposition 4.3. The MSE of the PTGLE based on W, LR and LM tests satisfies the following dominance relationship

$$
\begin{aligned}
& \operatorname{MSE}\left(\hat{\phi}_{L M}^{P T G L}(D)\right) \leq \operatorname{MSE}\left(\hat{\phi}_{L R}^{P T G L}(D)\right) \leq \operatorname{MSE}\left(\hat{\phi}_{W}^{P T G L}(D)\right) \quad \text { if } \quad d_{m i n} \in\left[d_{13}(\Delta, \alpha), 1\right) \text {, } \\
& \operatorname{MSE}\left(\hat{\phi}_{W}^{P T G L}(D)\right) \leq \operatorname{MSE}\left(\hat{\phi}_{L R}^{P T G L}(D)\right) \leq \operatorname{MSE}\left(\hat{\phi}_{L M}^{P T G L}(D)\right) \quad \text { if } \quad d_{\max } \in\left(0, d_{24}(\Delta, \alpha)\right]
\end{aligned}
$$

where $d_{13}(\Delta, \alpha)=\max \left\{d_{1}(\Delta, \alpha), d_{3}(\Delta, \alpha)\right\}$ and $d_{24}(\Delta, \alpha)=\min \left\{d_{2}(\Delta, \alpha), d_{4}(\Delta, \alpha)\right\}$

\section{Estimation of $D$}

To find an optimum value for biasing components in $D$, we consider the MSE function of $\tilde{\phi}(D)$ that can be expressed as

$$
\begin{gathered}
\operatorname{MSE}(\tilde{\phi}(D))=\sigma_{e}^{2} \sum_{i=1}^{p+q} \frac{\left(\lambda_{i}+d_{i}\right)^{2}}{\lambda_{i}\left(\lambda_{i}+1\right)^{2}}+\sum_{i=1}^{p+q} \frac{\phi_{i}^{*^{2}}\left(1-d_{i}\right)^{2}}{\left(\lambda_{i}+1\right)^{2}} \\
=\delta_{1}(D)+\delta_{2}(D) .
\end{gathered}
$$


For the generalized Liu estimator one needs to find values $d_{i}, i=1, \ldots, p+q$, so that the decrease in variance $\delta_{1}(D)$ is greater than the increase in the squared bias $\delta_{2}(D)$. In order to show that such values less than 1 exist, so that $\operatorname{MSE}(\tilde{\phi}(D))<\operatorname{MSE}(\tilde{\phi})$, we will take the derivative of equation (5.1) with respect to $d_{i}$ as

$$
\begin{aligned}
\frac{\partial \operatorname{MSE}(\tilde{\phi}(D))}{\partial d_{i}}= & 2 \sigma_{e}^{2} \sum_{i=1}^{p+q} \frac{\left(\lambda_{i}+d_{i}\right)}{\lambda_{i}\left(\lambda_{i}+1\right)^{2}}-2 \sum_{i=1}^{p+q} \frac{\phi_{i}^{*^{2}}\left(1-d_{i}\right)}{\left(\lambda_{i}+1\right)^{2}} \\
& \leq 2 \sigma_{e}^{2} \sum_{i=1}^{p+q} \frac{\left(\lambda_{i}+d_{\text {max }}\right)}{\lambda_{i}\left(\lambda_{i}+1\right)^{2}}-2 \sum_{i=1}^{p+q} \frac{\phi_{i}^{*^{2}}\left(1-d_{\text {max }}\right)}{\left(\lambda_{i}+1\right)^{2}}
\end{aligned}
$$

For $d_{i}=1, i=1, \ldots, p+q$, in (5.2), we obtain,

$$
\frac{\partial \operatorname{MSE}(\tilde{\phi}(D))}{\partial d_{i}}=2 \sigma_{e}^{2} \sum_{i=1}^{p+q} \frac{1}{\lambda_{i}\left(\lambda_{i}+1\right)}>0, \quad \text { since } \quad \lambda_{i} \geq 0
$$

Therefore, there exist values of $d_{i}\left(0<d_{i}<1\right)$, such that $\operatorname{MSE}(\tilde{\phi}(D))<\operatorname{MSE}(\tilde{\phi})$. Now from (5.2), the MSE of $\tilde{\phi}(D)$ is minimized at

$$
d_{\text {max }}=\frac{\sum_{i=1}^{p+q} \frac{\left(\phi_{i}^{x^{2}}-\sigma_{e}^{2}\right)}{\left(\lambda_{i}+1\right)^{2}}}{\sum_{i=1}^{p+q} \frac{\left(\sigma_{e}^{2}+\lambda_{i} \phi_{i}^{2}\right)}{\lambda_{i}\left(\lambda_{i}+1\right)^{2}}}
$$

Replacing $\phi_{i}^{*^{2}}$ and $\sigma_{e}^{2}$ by their unbiased estimate $\tilde{\phi}_{i}^{*^{2}}-\frac{\tilde{\sigma}_{e}^{2}}{\lambda_{i}}$ and $\tilde{\sigma}_{e}^{2}$, we obtain the optimum value of $d_{\text {max }}$ as

$$
d_{o p t}=1-\tilde{\sigma}_{e}^{2}\left\{\frac{\sum_{i=1}^{p+q} \frac{1}{\lambda_{i}\left(\lambda_{i}+1\right)}}{\sum_{i=1}^{p+q} \frac{\tilde{\phi}_{i}^{* 2}}{\left(\lambda_{i}+1\right)^{2}}}\right\} .
$$

It should be noted since $d_{\text {opt }}$ must rely on $(0,1)$, as $\tilde{\sigma}_{e}^{2}$ may become very large, a restriction must be put in obtaining $d_{\text {opt }}$ in numerical evaluations. 


\section{Numerical Illustrations}

In this section, to verify the theoretical results, first a Monte Carlo simulation is conducted to compare the PTGL estimators based on the W, LR and LM tests. In addition, performance analysis of the estimators is carried out using the well-known Portland cement dataset (Woods et al. (1932)).

\subsection{Monte Carlo Simulation}

The Monte Carlo simulation on the PTGL estimators based on W, LR and LM tests, is performed by considering different levels of multicollinearity. First, we generate the explanatory variables according to

$$
x_{i j}=\left(1-\rho^{2}\right)^{\frac{1}{2}} z_{i j}+\rho z_{i p}, \quad, i=1, \ldots, n, \quad j=1, \ldots, p .
$$

where $z_{i j}$ are independent standard normal pseudo-random numbers and $\rho^{2}$ is the correlation between any two explanatory variables. To achieve weekly, strong or severely collinear cases, $\rho$ is defined by $\rho \in\{0.6,0.7,0.9,0.99\}$. The response variable is generated by

$$
y_{i}=\sum_{j=1}^{p} \beta_{j} x_{i j}+e_{i}, \quad i=1, \ldots, n,
$$

where $e_{i}$ are independent multivariate student $\mathrm{t}$-distribution with $v=5$ degrees of freedom and $\beta_{j}$ are regression coefficients, where $\beta=(1,2,1,-1)^{T}$. We set $p=4$, $n=100$ and considered two stochastic linear restrictions in which $r_{1}=R_{1} \beta+\gamma_{1}$ and $r_{2}=R_{2} \beta+\gamma_{2}$, where $\gamma_{1}$ and $\gamma_{2}$ generated according to multivariate student $\mathrm{t}$ distributions with $v_{1}=v_{2}=5$ degree of freedom, considering

$$
R_{1}=\left[\begin{array}{llll}
0 & 1 & -1 & 1
\end{array}\right], \quad R_{2}=\left[\begin{array}{llll}
1 & 0 & 2 & 1
\end{array}\right] .
$$

The QB and MSE functions of the PTGL estimators based on W, LR and LM tests were computed with optimal biasing parameter $d_{\text {opt }}$. The results are tabulated in Tables 1 \& 2. It can be realized that by increasing the levels of multicollinearity, the estimated QB values of the PTGL estimators increase. The PTGL estimator based on W test has the smallest estimated QB value followed by the LR and the LM tests, which agree with theoretical results. For different significance levels $\alpha$, the PTGL estimator based on the LM test has the smallest estimated MSE value, followed by the LR and the W tests. 
Table 1: Estimated QB values of the PTGL estimators based on W, LR and LM tests using $d_{\text {opt }}$.

\begin{tabular}{l|cccccccc|}
\hline test $\backslash \alpha$ & & 0.015 & 0.02 & 0.025 & 0.05 & 0.1 & 0.15 & 0.2 \\
\hline \hline \multirow{4}{*}{$\mathrm{W}$} & $\rho=0.6$ & 0.2827 & 0.1946 & 0.1597 & 0.2309 & 0.0567 & 0.0130 & 0.0162 \\
& $\rho=0.7$ & 0.2337 & 0.1497 & 0.3019 & 0.1186 & 0.0972 & 0.0519 & 0.0140 \\
& $\rho=0.9$ & 0.3031 & 0.3066 & 0.1422 & 0.1211 & 0.0833 & 0.1191 & 0.0304 \\
& $\rho=0.99$ & 0.6020 & 0.5416 & 0.6198 & 0.4812 & 0.4756 & 0.3713 & 0.2415 \\
\hline \multirow{4}{*}{$\mathrm{LR}$} & $\rho=0.6$ & 0.2827 & 0.2103 & 0.1654 & 0.2309 & 0.0567 & 0.0130 & 0.0162 \\
& $\rho=0.7$ & 0.2337 & 0.1630 & 0.3150 & 0.1186 & 0.0973 & 0.0519 & 0.0140 \\
& $\rho=0.9$ & 0.3031 & 0.3066 & 0.1465 & 0.1284 & 0.0872 & 0.1191 & 0.0304 \\
& $\rho=0.99$ & 0.6075 & 0.5804 & 0.6461 & 0.4943 & 0.4820 & 0.3713 & 0.2415 \\
\hline \multirow{4}{*}{$\mathrm{LM}$} & $\rho=0.6$ & 0.2948 & 0.2331 & 0.1832 & 0.2398 & 0.0605 & 0.0130 & 0.0162 \\
& $\rho=0.7$ & 0.2819 & 0.1630 & 0.3239 & 0.1225 & 0.1030 & 0.0519 & 0.0140 \\
& $\rho=0.9$ & 0.3034 & 0.3175 & 0.1518 & 0.1329 & 0.0872 & 0.1191 & 0.0304 \\
& $\rho=0.99$ & 0.6249 & 0.5948 & 0.6614 & 0.5015 & 0.4827 & 0.3713 & 0.2415 \\
\hline \hline
\end{tabular}

\subsection{Portland Cement Data}

The Portland cement data obtained from an experimental study where it was used to measure the heat provided by chemical reactions in a type of cement. Typically, cement is a mixture of four components, where the ratio of each component affects the heat produced during the hardening stage. Design matrix and vector of responses are given by

$$
X=\left[\begin{array}{cccc}
7 & 26 & 6 & 60 \\
1 & 29 & 15 & 52 \\
11 & 56 & 8 & 20 \\
11 & 31 & 8 & 47 \\
7 & 52 & 6 & 33 \\
11 & 55 & 9 & 22 \\
3 & 71 & 17 & 6 \\
1 & 31 & 22 & 44 \\
2 & 54 & 18 & 22 \\
21 & 47 & 4 & 26 \\
1 & 40 & 23 & 34 \\
11 & 66 & 9 & 12 \\
10 & 68 & 8 & 12
\end{array}\right], \quad y=\left[\begin{array}{c}
78.5 \\
74.3 \\
104.3 \\
87.6 \\
95.9 \\
109.2 \\
102.7 \\
72.5 \\
93.1 \\
115.9 \\
83.8 \\
113.3 \\
109.4
\end{array}\right]
$$


Table 2: Estimated MSE values of the PTGL estimators based on W, LR and LM tests using $d_{\text {opt }}$.

\begin{tabular}{l|cccccccc|}
\hline test $\backslash \alpha$ & & 0.015 & 0.02 & 0.025 & 0.05 & 0.1 & 0.15 & 0.2 \\
\hline \hline \multirow{4}{*}{$\mathrm{W}$} & $\rho=0.6$ & 2.5818 & 2.5260 & 2.9051 & 3.0065 & 4.3675 & 4.1738 & 3.3850 \\
& $\rho=0.7$ & 3.3948 & 2.7998 & 3.0899 & 3.3781 & 4.2935 & 4.5594 & 3.4010 \\
& $\rho=0.9$ & 3.7536 & 3.8481 & 3.4213 & 3.5717 & 3.7685 & 3.7890 & 5.9117 \\
& $\rho=0.99$ & 4.1195 & 4.4835 & 4.3354 & 4.9998 & 4.7253 & 5.9850 & 5.7964 \\
\hline \multirow{4}{*}{$\mathrm{LR}$} & $\rho=0.6$ & 2.4848 & 2.5260 & 2.8819 & 2.9912 & 4.3675 & 4.1738 & 3.3850 \\
& $\rho=0.7$ & 3.3948 & 2.7682 & 3.0893 & 3.3648 & 4.2935 & 4.5594 & 3.4010 \\
& $\rho=0.9$ & 3.7409 & 3.8024 & 3.4050 & 3.5344 & 3.7685 & 3.7783 & 5.9117 \\
& $\rho=0.99$ & 4.1099 & 4.4197 & 4.2882 & 4.9998 & 4.7253 & 5.9850 & 5.7964 \\
\hline \multirow{4}{*}{$\mathrm{LM}$} & $\rho=0.6$ & 2.4327 & 2.4974 & 2.8098 & 3.0065 & 4.3675 & 4.1738 & 3.3863 \\
& $\rho=0.7$ & 3.3773 & 2.7320 & 3.0234 & 3.3396 & 4.2935 & 4.5594 & 3.4084 \\
& $\rho=0.9$ & 3.6145 & 3.7781 & 3.3530 & 3.5150 & 3.7685 & 3.7890 & 5.9117 \\
& $\rho=0.99$ & 4.0838 & 4.4835 & 4.3354 & 4.9931 & 4.7253 & 5.9850 & 5.8025 \\
\hline \hline
\end{tabular}

The ordinary least squares estimator of $\beta$ and $\sigma^{2}$ are obtained as

$$
\tilde{\beta}=(2.1930,1.1533,0.7585,0.4863)^{T}, \quad \tilde{\sigma}^{2}=4.0468 .
$$

Arashi et al. (2015) criticized the assumption of normality in this example and suggested to use heavier tailed alternatives such as Cauchy or $\mathrm{t}$ - models. Under a t-model, we consider the stochastic restriction $r=R \beta+\gamma$, where $\gamma$ is generated from a multivariate student $\mathrm{t}$-distribution with $v=5$ degrees of freedom, with $R=\left[\begin{array}{llll}1 & -1 & 1 & 0\end{array}\right]$. In order to compare the three PTGL estimators based on W, LR and LM tests, we computed the root mean squared prediction error (RMPE). The results are provided in Table 3 for different significance levels and $d_{\text {opt }}$. It can be seen that for small values $\alpha$, the PTGL estimator based on the LM test has the smallest RMPE value, followed by the LR and the $\mathrm{W}$ tests, and when $\alpha=0.2$, RMPE values of the PTGLEs based on W, LR and LM tests are the same.

Table 3: Estimated RMPE values of the PTGL estimators based on W, LR and LM tests.

\begin{tabular}{l|ccccccc|}
\hline test $\backslash \alpha$ & 0.015 & 0.02 & 0.025 & 0.05 & 0.1 & 0.15 & 0.2 \\
\hline \hline $\mathrm{W}$ & 45.1965 & 57.0744 & 49.5683 & 48.2168 & 59.2755 & 54.5541 & 60.7536 \\
\hline $\mathrm{LR}$ & 45.1659 & 57.0589 & 49.5603 & 48.2138 & 59.2696 & 54.5469 & 60.7536 \\
\hline $\mathrm{LM}$ & 42.9257 & 56.6578 & 49.3937 & 48.2055 & 59.2539 & 54.5468 & 60.7536 \\
\hline \hline
\end{tabular}




\section{Conclusions}

In this paper, we have studied the effect of three large tests, namely, the W, LR and LM tests on the performance of the PTGL estimator for estimating the regression parameters, when there are a series of linear stochastic restrictions available about the regression parameters. For the generalized Liu estimator the optimum biasing matrix is obtained and extensive numerical studies carried out to evaluate the performance of PTGL estimator. Through numerical illustrations, we achieved for different significance levels $\alpha$ and $d_{\text {opt }}$, the PTGL estimator based on the LM test that has the smallest MSE value followed by the LR and $\mathrm{W}$ tests. Hence, we recommend the practitioner to use the LM test, dealing with multicollinear situations, for estimating the regression parameters.

\section{Acknowledgements}

The authors would like to thank the Associate Editor and two anonymous reviewers for constructive comments which led to adding more details in the paper and a great deal of improvements in the presentation.

\section{References}

Akdeniz, F. and Kaciranlar, S. (1995), On the almost unbiased generalized Liu estimator and unbiased estimation of the Bias and MSE. Communications in Statistics-Theory and Methods, 24(7), 1789-1797.

Anderson, T. W. (2003), An Introduction to Multivariate Statistical Analysis. 3rd Ed., John Wiley, New York.

Arashi, M., Janfada, M. and Norouzirad, M. (2015), Singular ridge regression with stochastic constrains. Communications in Statistics-Theory and Methods, 44, 1281-1292.

Bancroft, T. A. (1944), On biases in estimation due to use of preliminary tests of significance. The Annals of Mathematical Statistics, 15, 190-204.

Karbalaee, M. H., Arashi, M. and Tabatabaey, S. M. M. (2017), Performance analysis of the preliminary test estimator with series of stochastic restrictions. Communications in Statistics-Theory and Methods, DOI:10.1080/03610926.2017.1300275. 
Khan, S., Saleh, A. K. Md. Ehsanes (1997), Shrinkage pre-test estimator of the intercept parameter for a regression model with multivariate student-t errors. Biometrical Journal, 39, 1-17.

Kibria, B. M. G. (1996), On shrinkage ridge regression estimators for restricted linear models with multivariate $t$ disturbances. Students, 1(3), 177-188.

Kibria, B. M. G. (2003), Performance of some new ridge regression estimators. Communications in Statistics-Simulation and Computation, 32, 419-435.

Kibria, B. M. G. and Saleh, A. K. Md. Ehsanes (2004), Preliminary test ridge regression estimators with student's t errors and conflicting test-statistics. Metrika, 59, 105-124.

Kuran, O and Ozkale, M. R. (2016), Gilmour's approach to mixed and stochastic restricted ridge predictions in linear mixed models. Linear Algebra and its Applications, $508,22-47$.

Li, Y. and Yang, H. (2010), A new ridge-type estimator in stochastic restricted linear regression. Statistics, 45, 123-130.

Liu, K. (1993), A new class of biased estimate in linear regression. Communications in Statistics-Theory and Methods, 22, 393-402.

Ozkale, M. R. (2009), A stochastic restricted ridge regression estimator. Journal of Multivariate Analysis, 100, 1706-1716.

Roozbeh, M. (2015), Shrinkage ridge estimators in semi parametric regression models. Journal of Multivariate Analysis, 136, 56-74.

Saleh, A. K. Md. Ehsanes. (2006), Theory of Preliminary Test and Stein-Type Estimation with Application. John Wiley, New York.

Saleh, A. K. Md. Ehsanes and Sen, P. K. (1978), Non-parametric estimation of location parametric after a preliminary test regression. The Annals of Statistics, 6, 154-168.

Tabatabaey, S. M. M., Saleh, A. K. Md. Ehsanes and Kibria, B. M. G. (2004), Estimation strategies for parameters of the linear regression model with spherically symmetric distributions. J. Statist. Res., 38(1), 13-31.

Ullah, A. and Zinde-Walsh, V. (1984), On the robustness of LM, LR and W Tests in Regression Model. Econometrica, 52, 1055-1066.

Woods, H., Steinour, H. and Starke, H. R. (1932), Effect of composition of Portland cement on heat evolved during hardening. Industrial and Engineering Chemistry, 24, 1207-1241. 
Yang, H., Chang, X. F. and Liu, D. (2009), Improvement of the Liu estimator in weighted mixed regression. Communications in Statistics-Theory and Methods, 38, 285-292.

Yang H. and Xu J. (2011), Preliminary test Liu estimators based on the conflicting W, LR and LM tests in a regression model with multivariate student-t error. Metrika, 73(3), 275-292.

Yang H. and $\mathrm{Xu} \mathrm{J.} \mathrm{(2009),} \mathrm{An} \mathrm{alternative} \mathrm{stochastic} \mathrm{restricted} \mathrm{Liu} \mathrm{estimator} \mathrm{in} \mathrm{linear}$ regression. Statistical Papers, 50(3), 639-647.

Yang, H. and Wu, J. (2012), A stochastic restricted k-d class estimator. Statistics, 46(6), 759-766.

Yuzbasi, B., Asar, Y., Sik, S. M., and Demiralp, A. (2018), Improving estimations in quantile regression model with autoregressive errors, Thermal Science, http://doi.org/10.2298/TSCI170612275Y.

Yuzbasi, B., Ahmed, S. E., and Aydin, D. (2017), Ridge-type pretest and shrinkage estimations in partially linear models. Statistical Papers, http://doi.org/10.1007/s00362017-0967-8.

Wu, J. and Asar, Y. (2017), A weighted stochastic restricted ridge estimator in partially linear model. Communications in Statistics-Theory and Methods, 46(18), 9274-9283.

Zellner, A. (1962), An efficient method of estimating seemingly unrelated regressions and tests for aggregation bias. Journal of the American statistical Association, 57(298), 348-368. 
